\title{
The spatiotopic representation of visual objects across time
}

\author{
Thérèse Collins ${ }^{1}$
}

Published online: 27 June 2016

(C) The Psychonomic Society, Inc. 2016

\begin{abstract}
Each eye movement introduces changes in the retinal location of objects. How a stable spatiotopic representation emerges from such variable input is an important question for the study of vision. Researchers have classically probed human observers' performance in a task requiring a location judgment about an object presented at different locations across a saccade. Correct performance on this task requires realigning or remapping retinal locations to compensate for the saccade. A recent study showed that performance improved with longer presaccadic viewing time, suggesting that accurate spatiotopic representations take time to build up. The first goal of the study was to replicate that finding. Two experiments, one an exact replication and the second a modified version, failed to replicate improved performance with longer presaccadic viewing time. The second goal of this study was to examine the role of attention in constructing spatiotopic representations, as theoretical and neurophysiological accounts of remapping have proposed that only attended targets are remapped. A third experiment thus manipulated attention with a spatial cueing paradigm and compared transsaccadic location performance of attended versus unattended targets. No difference in spatiotopic performance was found between attended and unattended targets. Although only negative results are reported, they might nevertheless suggest that spatiotopic representations are relatively stable over time.
\end{abstract}

Thérèse Collins

therese.collins@parisdescartes.fr

1 Laboratoire Psychologie de la Perception, Université Paris Descartes \& CNRS (UMR 8242), 45 rue des Saints-Pères, 75006 Paris, France

\section{Introduction}

Visual perception starts in the eye, with the retinal image of the outside world. This retinal image changes several times a second as we move our eyes around to project regions or objects of interest onto the high-resolution fovea. Despite the constant changes in the input, our phenomenological impression of the visual world is that of stable, object-centered coordinates. How such a spatiotopic representation of the visual world builds up across saccades is a matter of long-standing debate and has attracted a lot of recent interest from psychologists (Cavanagh, Hunt, Afraz \& Rolfs, 2010) and physiologists (Sommer \& Wurtz, 2008).

Researchers who measure spatiotopic representations in the laboratory often recourse to the classic in-flight displacement task, in which observers report in which direction a visual target was displaced during the saccade towards it (Bridgeman, Hendry \& Stark, 1975). Successfully performing this task requires comparing the actual post-saccadic location of the target with its predicted location, based on an internal estimate of the saccade (Collins, Rolfs, Deubel \& Cavanagh, 2009). Observers are notoriously bad at this task, suggesting that their spatiotopic representation of target location is poor (Bridgeman et al., 1975). However, several simple experimental manipulations drastically increase performance and reveal the mechanisms involved in constructing a spatiotopic representation. For example, inserting a blank period between saccade landing and post-saccadic target reappearance leads to almost perfect performance (Deubel, Schneider \& Bridgeman, 1996; Deubel, Bridgeman \& Schneider, 1998; Deubel, 2004). Adding an irrelevant orthogonal component to the displacement similarly improves performance (Wexler \& Collins, 2014). Finally, small improvements are observed when the displacement is accompanied by a change of visual features (Demeyer, De Graef, Wagemans \& Verfaillie, 2010). These studies suggest 
that accurate spatiotopic representations are achieved by the visual system but that there is a tolerance for small discrepancies between predicted and actual target locations, probably to compensate for low-level motor noise (Niemeier, Crawford \& Tweed, 2003). They also suggest that the two main contributors to spatiotopic representations are the presence of a target immediately after a saccade and the accuracy of the internal representation of the saccade, while the target's visual features play a lesser role.

The internal representation of the saccade (corollary discharge, efference copy) is what allows the alignment of target representations in space across retinal displacements. Such a process has been termed remapping (Cavanagh et al., 2010). Indeed, if a saccade causes the retinal image to shift, metric information about the saccade can allow the visual system to attribute the retinal displacement to self-movement and to successfully localize the visual object in outside space. Remapping can be predictive, allowing a comparison between the predicted sensory consequences of the eye movement and its actual consequences, but it does not have to be: the realignment can also occur after the saccade.

Recently, Zimmermann, Morrone, and Burr (2013) reported that spatiotopic representations take time to build up. They asked observers to perform the classic in-flight displacement task but varied the time that the presaccadic target was available before the instruction to make a saccade. They reported that spatiotopic performance increased with the duration of presaccadic exposure and proposed that this meant that processes involved in constructing the spatiotopic representation of the target took time to develop.

The current study had three experiments and two goals: to replicate Zimmerman et al. (2013) and to examine the role of attention in remapping. All experiments were variations of the classic in-flight displacement task. The first experiment was an exact replication of the saccade condition in Zimmerman et al. (2013). The second experiment was a modified replication - one that used a slightly different design. The hypothesis for both of these replications (exact and modified) was that spatiotopic performance improves with longer pre-saccadic exposure. If spatiotopic representations do take time to build up, then the effect should withstand minor methodological changes and occur in the modified replication as well. Furthermore, replicating the effect with the modified design will aid the comparison the classic saccadic suppression of displacement studies that used that design (Bridgeman et al., 1975; Deubel et al., 1996).

The second goal was to examine the role of attention in remapping. If visual information were remapped with every saccade, the sheer computational (and neuronal) demands would be excessively large. Instead, researchers have proposed that only a few items are remapped: those to which attention has been drawn (Cavanagh et al., 2010; Rolfs et al., 2011; Joiner, Cavanaugh \& Wurtz, 2011; Jonikaitis,
Szinte, Rolfs \& Cavanagh, 2013). The third experiment thus sought to measure spatiotopic performance while manipulating the attention drawn to the pre-saccadic target. The hypothesis was that performance would be better for attended targets relative to nonattended targets.

\section{Experiment 1: Replication}

\section{Subjects}

Eight subjects were recruited from the French RISC subject database (Réseau d'Informations sur les Sciences Cognitives). The number of subjects was chosen to match the power of the original study (Zimmerman et al. 2013). Inclusion criteria were: aged 20-35 years; corrected or corrected-to-normal vision; no self-reported neurological or cognitive impairments; no current medication susceptible of impinging upon attention or vigilance. All subjects received $10 €$ /hour compensation. The local ethics board (CERES, Université Paris Descartes) approved the experiments.

\section{Instruments and stimuli}

Stimuli were $0.2^{\circ}$ diameter dots presented on a 2200 Formac ProNitron 22,800 screen with a resolution of 1,024 by 768 pixels and a refresh rate of $100 \mathrm{~Hz}$. Subjects were seated $57 \mathrm{~cm}$ from the screen and their heads kept stable by chin- and forehead-rests. The fixation dot was presented $10^{\circ}$ left (or right) of screen center. The saccade target was presented $20^{\circ}$ to the right (or left) of fixation. The direction of saccades was fixed for a given experimental session. During saccade execution, the target dot stepped to one of 15 equiprobable locations $\left( \pm 2^{\circ}, \pm 1.5^{\circ}, \pm 1.25^{\circ}, \pm 1^{\circ}, \pm 0.75^{\circ}, \pm 0.5^{\circ}, \pm 0.25^{\circ}, 0^{\circ}\right.$ relative to the initial position; Fig. 1). The displaced target dot remained on screen until subjects gave a response. All experiments and analyses were performed with Matlab (version R2014b) using the Psychophysics Toolbox extensions (Brainard, 1997; Pelli, 1997; Kleiner et al., 2007).

\section{Eye movement recording and analysis}

Viewing was binocular and movements of the right eye were monitored with an Eyelink $1 \mathrm{k}$ (SR Research, Mississauga, Ontario, Canada) at 1,000-Hz sampling rate. At the beginning of a session, the Eyelink was calibrated with the standard ninepoint Eyelink procedure. Before each trial, fixation was checked. If the distance between the fixation check and the calibration was greater than $1.5^{\circ}$, a new calibration was initiated. Calibration also was automatically renewed every 100 trials. Online saccade detection was based on a boundary criterion: gaze-contingent changes occurred when the eye position crossed one fourth of the target eccentricity. Eye movement 
a) Stimuli

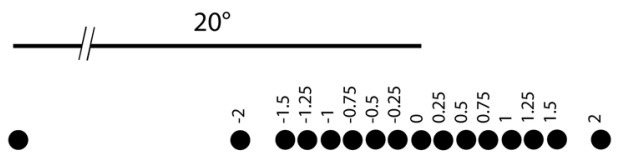

b) Procedure

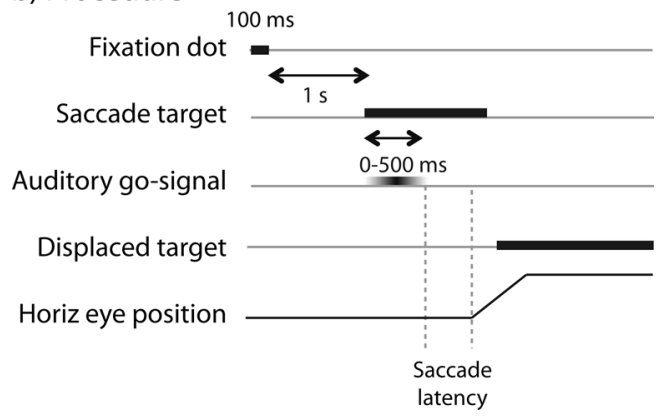

Fig. 1 In-flight displacement task for Experiment 1. a Stimuli. The saccade target appeared $20^{\circ}$ to the left or right, and was displaced during the saccade $\left( \pm 2^{\circ}, \pm 1.5^{\circ}, \pm 1.25^{\circ}, \pm 1^{\circ}, \pm 0.75^{\circ}, \pm 0.5^{\circ}, \pm 0.25^{\circ}, 0^{\circ}\right.$ relative to the initial position). $\mathbf{b}$ Procedure. The fixation dot appeared for $100 \mathrm{~ms}$ followed by a $1,000-\mathrm{ms}$ blank screen. The saccade target dot appeared and subjects had to wait 0 to $500 \mathrm{~ms}$ for the auditory gosignal to perform a saccade to the target. During saccade execution, the target was displaced to another location

traces were subsequently analyzed offline. Instantaneous velocity and acceleration were computed for each data sample and compared to a threshold $\left(30 \% \mathrm{~s}\right.$ and $\left.8000 \% / \mathrm{s}^{2}\right)$. Saccade onset was defined as two consecutive above-threshold samples for both criteria. Saccade offset was defined as the beginning of the next 20-ms period of below threshold samples.

\section{Procedure}

Subjects were instructed to fixate the dot, which remained on screen for $100 \mathrm{~ms}$. It then disappeared for 1,000 ms during which subjects were to maintain fixation at the remembered fixation dot location. After the 1-sec delay, the target dot appeared. Subjects were to withhold saccading to this target until instructed to do so by a beep played 0 to $500 \mathrm{~ms}$ later. This delay allowed the preexposure duration to vary. During saccade execution, the target stepped to one of the possible new locations (Fig. 1). Subjects reported whether the target stepped to the left or to the right by pressing on one of two keyboard buttons. Each subject performed 2,430 trials, divided into three approximately 1.5-hour long sessions. Each displacement size was tested 162 times.

\section{Analyses}

Usual saccade parameters were analyzed (latency and amplitude gain). First, saccade latency (ms) was defined as the delay between the go-signal to make a saccade (beep) and saccade onset. The influence of overlap delay on saccade latency and amplitude (gain: ratio of saccade amplitude to target eccentricity) was analyzed by regressing each dependent variable on overlap. The hypothesis was that neither latency nor amplitude would depend on overlap.

By summing saccade latency and overlap delay, the pre-exposure duration was calculated for each trial. Each subject's pre-exposure distribution was divided into three equal thirds, defined as "short," "medium," and "long" pre-exposure durations. Perceptual performance was analyzed by fitting individual cumulative gaussians to the proportion "forwards" responses. The difference in displacement size between the $50 \%$ and $75 \%$ points on the psychometric curve represented the perceptual threshold, which was reported as a Weber fraction of saccade amplitude. Trials were labeled according to their pre-exposure duration and psychometric functions fit separately for the three categories. The hypothesis was that threshold would decrease with pre-exposure duration, replicating Zimmerman et al. (2013).

All raw data are available via Figshare.

\section{Results}

Trials were discarded if saccade latency or amplitude were more than 2 standard deviations from the mean (calculated without that trial). Overall, $87 \%$ of trials were included in the analyses. Mean saccade latency was slightly long for visually guided saccades ( $437 \pm 54 \mathrm{~ms}$; mean \pm standard deviation), probably due to the auditory go-signal. Mean saccade gain was $0.91 \pm 0.04$. Saccade latency depended on overlap duration (linear regression on individual data, all $p s<0.005$ ): the longer the overlap duration, the shorter the latency, although the effect was small (average slope $-0.10 \mathrm{~ms}$ ). Saccade amplitude, on the other hand, did not depend on overlap duration (all individual $p>0.3$ ).

Overlap and latency were summed into pre-exposure duration, and perceptual performance was analysed for short, medium, and long durations. Average durations for the three categories were $605 \pm 49 \mathrm{~ms}, 754 \pm 61 \mathrm{~ms}$, and 1,252 \pm $121 \mathrm{~ms}$. Thresholds did not depend on pre-exposure duration $(\mathrm{F}<1)$. Binning of pre-exposure into thirds in this way may have masked the effect because the windows were similar in average pre-exposure duration and also relatively long. To further analyse the effect of pre-exposure duration, trials were binned into six preexposure windows (average durations $524 \pm 50,605 \pm 49,677 \pm 55,758 \pm 62,854 \pm 59,1$, $252 \pm 121 \mathrm{~ms})$, but this again failed to reveal an effect $(\mathrm{F}<$ 1). Finally, because pre-exposure duration was relatively long, the shortest third or sixth might already afford sufficient time for spatiotopic representations to develop, and so preexposure duration was binned - with an unequal number of trials per bin — as $<300 \mathrm{~ms}$ (average number of trials per participant $=31), 300-600 \mathrm{~ms}(\mathrm{n}=662), 600-900 \mathrm{~ms}(\mathrm{n}=1175)$, and $>900 \mathrm{~ms}(\mathrm{n}=249)$. However, again no effect of 
pre-exposure duration on perceptual performance emerged ( $\mathrm{F}<1$; Figs. 2a and $\mathrm{b}$ ). (A post-hoc analysis also found no changes in bias across pre-exposure durations).

\section{Experiment 2: Modification}

\section{Subjects and power analysis}

To estimate the number of subjects needed to reveal the perceptual effect, values reported by Zimmermann et al. (2013) were used. The perceptual threshold (SD of the psychometric function) for the shortest pre-exposure duration was $1.14^{\circ}$, the perceptual threshold for the longest pre-exposure duration was $0.66^{\circ}$, and the standard error was approximately $0.2^{\circ}$. To detect a similar effect with a power of $0.90,15$ subjects are necessary. Increasing the number to 20 gives a power of 0.97 .

Twenty subjects were recruited from the French RISC subject database (Réseau d'Informations sur les Sciences Cognitives). Inclusion criteria and compensation were identical to Experiment 1. Three subjects were unable to perform the task (flat psychometric functions) and so were removed from the analyses, which gives a final power of 0.93 .

\section{Instruments and stimuli}

Identical to experiment 1 , except that the saccade target was located $12^{\circ}$ from the fixation point.

\section{Eye movement recording and analysis}

Identical to experiment 1 .

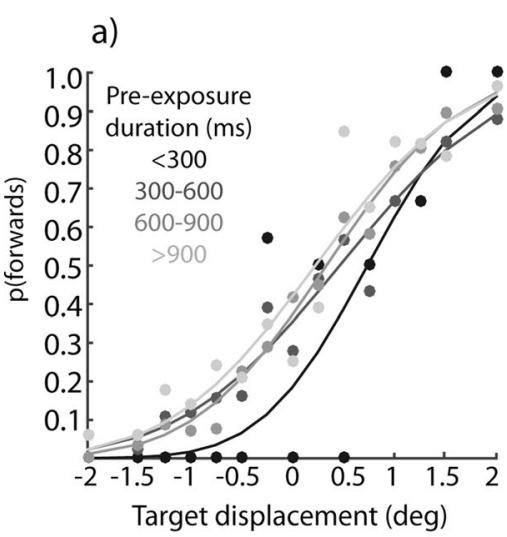

Fig. 2 Results. a Example psychometric functions for an individual subject in Experiment 1. b Mean threshold and $95 \%$ confidence intervals (bootstraps) as a function of pre-exposure duration in

\section{Procedure}

Subjects were instructed to fixate a red fixation dot. When correct fixation was detected, the fixation dot turned black and at the same time, the saccade target appeared. Both fixation dot and saccade target stayed on for an overlap delay of 100 to $800 \mathrm{~ms}$. After the overlap delay, the fixation dot disappeared and subjects made a saccade to the target. During saccade execution, the target stepped to one of the possible new locations (Fig. 3). Subjects reported whether the target stepped to the left or to the right by pressing on one of two keyboard buttons. Each subject performed 2,430 trials, divided into 3 approximately 1.5-hour long sessions. Each displacement size was tested 162 times.

\section{Analyses}

Identical to Experiment 1, except that saccade latency was the delay between the offset of the fixation dot and the onset of the saccade.

\section{Results}

Trials were discarded if saccade latency or amplitude were more than 2 standard deviations from the mean (calculated without that trial). Overall, $89 \%$ of trials were included in the analyses. Both saccade latency and amplitude gain were normal for visually guided saccades $(283 \pm 34 \mathrm{~ms}$ and $0.93 \pm$ $0.06 \mathrm{deg}$ ) and did not depend on exposure duration (linear regressions; amplitude: all individual $p>0.15$; latency: all individual $p>0.06$ except three subjects with a significant correlation, $0.001>p>0.03$ ).

As for Experiment 1, overlap and latency were summed into pre-exposure duration. Thresholds did not vary with

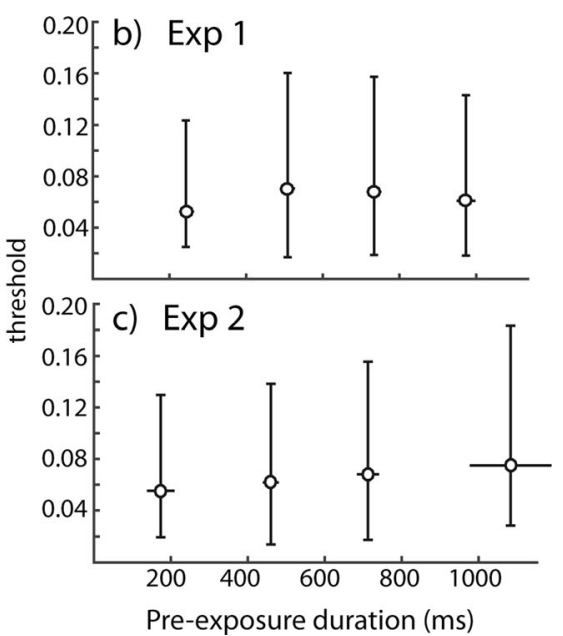

Experiment 1. Most of the horizontal error bars are smaller than the markers. c Mean threshold and $95 \%$ confidence intervals (bootstraps) as a function of pre-exposure duration in Experiment 2 
a) Stimuli

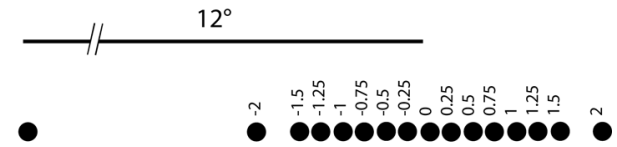

b) Procedure

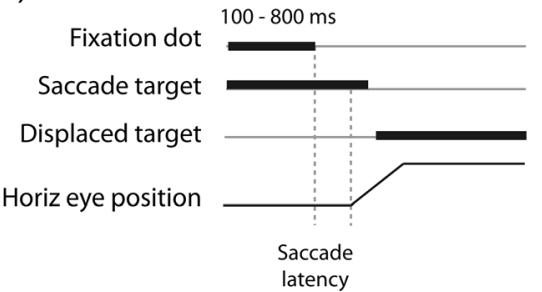

Fig. 3 In-flight displacement task for Experiment 2. a Stimuli. The saccade target appeared $12^{\circ}$ to the left or right and was subsequently displaced $\left( \pm 2^{\circ}, \pm 1.5^{\circ}, \pm 1.25^{\circ}, \pm 1^{\circ}, \pm 0.75^{\circ}, \pm 0.5^{\circ}, \pm 0.25^{\circ}, 0^{\circ}\right.$ relative to the initial position). b Procedure. The fixation and target dots appeared simultaneously and overlapped for 100 to $800 \mathrm{~ms}$. Fixation offset was the go-signal for the saccade, and during saccade execution, the target was displaced to another location

pre-exposure duration, whatever the number of windows: short, medium, and long (average durations $443 \pm 34 \mathrm{~ms}$, $561 \pm 95 \mathrm{~ms}$, and $1,942 \pm 873 \mathrm{~ms} ; \mathrm{F}<1)$; six windows of equal number of trials $(399 \pm 24,443 \pm 34,491 \pm 53,566 \pm 96$, $698 \pm 139,1942 \pm 873 \mathrm{~ms} ; \mathrm{F}<1)$; four windows of unequal number of trials $(<300 \mathrm{~ms}$ (average number of trials per participant $=123) 300-600 \mathrm{~ms}(\mathrm{n}=1,485), 600-900 \mathrm{~ms}(\mathrm{n}=403)$, $>900 \mathrm{~ms}(\mathrm{n}=158) ; \mathrm{F}<1$; Fig. $2 \mathrm{c})$.

\section{Experiment 3: Influence of attention on SSD}

\section{Subjects and power analysis}

Performing a power analysis for experiment 3 was not as simple as for experiment 2 , because there are no reports in the literature of the potential size of the attentional effect on spatiotopic performance. The number of subjects was therefore simply matched across experiments 2 and $3(n=20)$.

\section{Instruments and stimuli}

Instruments were identical to experiment 1 . Stimuli were $0.2^{\circ}$ diameter dots, a $1.5^{\circ}$-diameter circle outline (the cue), and a $1^{\circ}$ vertical bar on which a $0.1^{\circ}$ diameter dot was superimposed, either at the top or the bottom (the pretest target; Fig. 4).

\section{Procedure}

The procedure is illustrated in Fig. 4. In the pre-test, subjects performed a classic spatial cueing task. A red fixation dot appeared at screen center. Once correct fixation was detected, the red fixation dot turned black and 200-500 ms later, the cue appeared $8^{\circ}$ to the left or right for $250 \mathrm{~ms}$. After a delay of 100 or $600 \mathrm{~ms}$, the target appeared randomly to the left or to the right. Subjects had to report whether the dot was at the top or bottom of the vertical line. Cued trials were defined as trials on which the cue and the target were on the same side (as

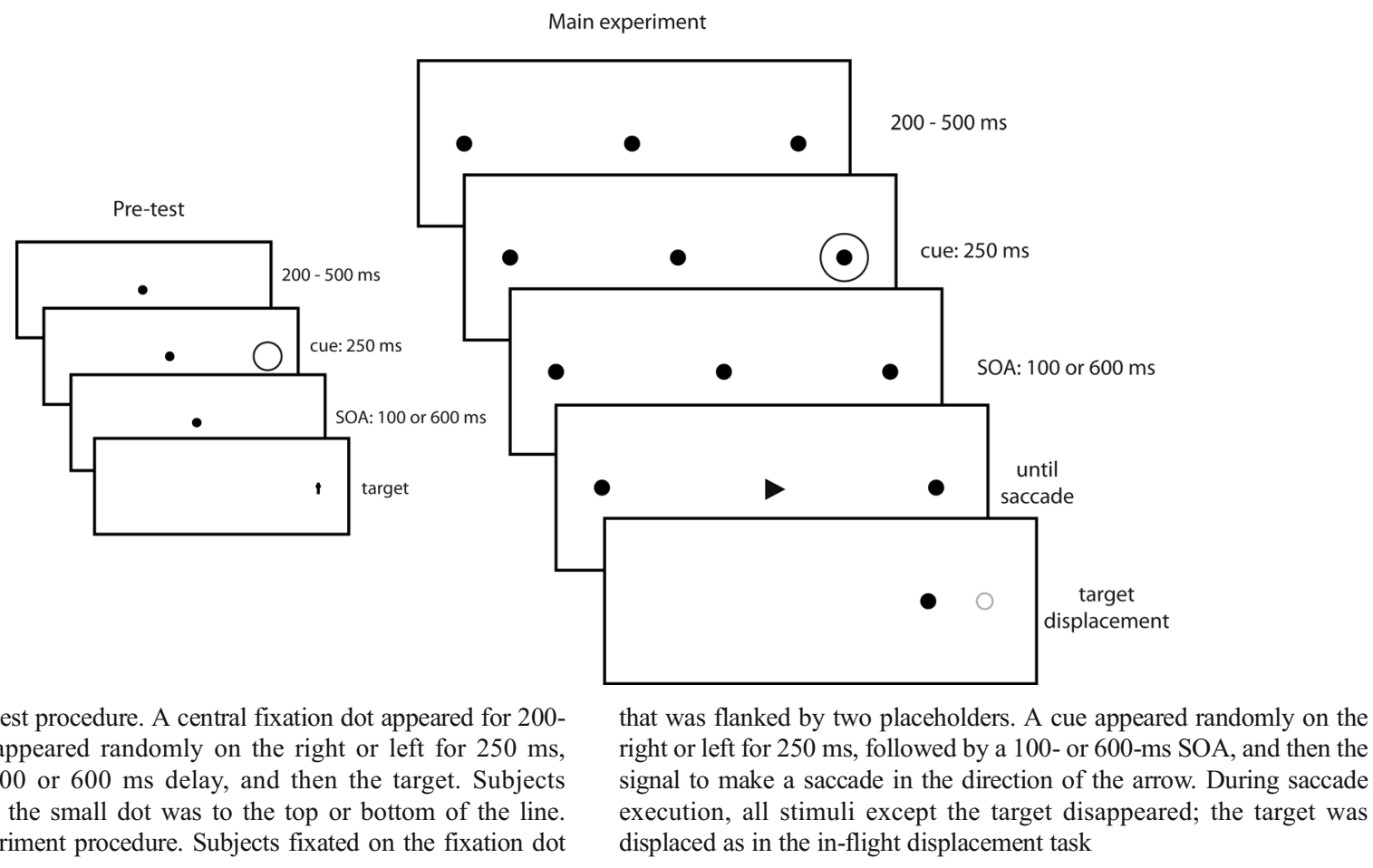

Fig. 4 Left: pre-test procedure. A central fixation dot appeared for 200$500 \mathrm{~ms}$. A cue appeared randomly on the right or left for $250 \mathrm{~ms}$, followed by a 100 or $600 \mathrm{~ms}$ delay, and then the target. Subjects reported whether the small dot was to the top or bottom of the line. Right: main experiment procedure. Subjects fixated on the fixation dot that was flanked by two placeholders. A cue appeared randomly on the , followed by a 100- or 600-ms SOA, and then the execution, all stimuli except the target disappeared; the target was displaced as in the in-flight displacement task 
illustrated in Fig. 4), and uncued trials when they were on opposite sides.

Success in the pre-test was a requirement to proceed to the experiment and was defined as a positive spatial cueing effect: better performance on the cued side relative to the uncued side. Performance here was both greater $\%$ correct and faster RT. Only the short SOA (100 ms) was taken into account for success, because at the longer SOA inhibition of return was likely to occur but was more difficult to measure. Because the pre-test was aimed only at examining whether the cue successfully oriented attention, obtaining inhibition of return was not a strict requirement.

In the experiment, subjects performed the classic in-flight displacement task as in experiment 2, with some additional steps. Each trial started with the presentation of a red fixation dot. Once correct fixation was detected, the fixation dot turned black and two placeholder dots appeared $8^{\circ}$ to the left and right. After a delay of 200-500 ms, the cue appeared to the left or the right, framing one of the placeholders, for $250 \mathrm{~ms}$. After an SOA of 100 or $600 \mathrm{~ms}$, the saccade target was indicated by a central arrow pointing left or right. In congruent trials, the arrow pointed to the side on which the cue had appeared. In non-congruent trials, the arrow pointed in the opposite direction. Subjects were free to saccade to the instructed side as soon as the arrow cue appeared. During saccade execution, the saccade target dot was displaced to one of seven equiprobable new locations $\left( \pm 1.5^{\circ}, \pm 1^{\circ}, \pm 0.5^{\circ}\right.$, $0^{\circ}$ from the initial position). Subjects had to report whether the target stepped to the left or right by pressing on one of two keyboard buttons.

Each subject performed 896 trials in one approximately 1.5-hour-long session. Each displacement was tested 28 times for each combination of congruency and SOA.

\section{Eye movement recording and analysis}

Identical to Experiment 1.

\section{Analyses}

Usual saccade parameters (latency and amplitude gain) were analyzed. First, saccade latency (ms) was defined as the delay between the go-signal to make a saccade (appearance of the arrow) and saccade onset. Saccade latency and amplitude in congruent versus non-congruent trials, and in SOA 100 versus SOA 600 conditions, were analyzed with a two-way ANOVA. The hypothesis was that saccade latency would be faster in congruent trials relative to non-congruent trials, but there would be no effect on saccade amplitude.

Perceptual performance was analyzed by fitting cumulative gaussians to the proportion "forwards" responses, separately for four conditions defined by the combination of SOA (100 vs. $600 \mathrm{~ms}$ ) and congruency (between cue side and saccade side). The difference in displacement size between the $50 \%$ and $75 \%$ points on the psychometric curve represented the perceptual threshold and reported as a Weber fraction of saccade amplitude. The hypothesis was that for the short SOA, thresholds would be smaller for congruent trials relative to non-congruent trials, whereas for the long SOA, inhibition of return would occur and thresholds would be smaller for non-congruent trials relative to congruent trials.

\section{Results}

Trials were discarded if saccade latency or amplitude were more than 2 standard deviations from the mean (calculated without that trial). Overall, $90 \%$ of trials were included in the analyses. Mean saccade latency $(341 \pm 50 \mathrm{~ms})$ and amplitude gain $\left(0.88 \pm 0.18^{\circ}\right)$ were within the normal range for visually guided saccades. Neither latency nor amplitude depended on cueing or SOA (all $\mathrm{F}<1$ ). Perceptual performance is plotted in Fig. 5. Mean performance and $95 \%$ confidence intervals were obtained by bootstrapping. There was no effect of cueing or of SOA.

\section{Discussion}

Two experiments investigated how spatiotopic performance depended on the duration for which the visual object was visible before the change in retinal coordinates. Both experiments failed to find any significant effect of pre-exposure duration: it was not the case that the longer the saccade target was present before the saccade, the better intrasaccadic target displacements were perceived, as previously reported (Zimmermann et al., 2013).

The rationale behind the modified or "conceptual" (Pashler \& Harris, 2012) replication (Experiment 2) was that if the slow build-up of spatiotopic information is a robust effect, it should withstand minor methodological changes. It did not. Several methodological details were different between the original study and experiment 2 , any of which could explain the

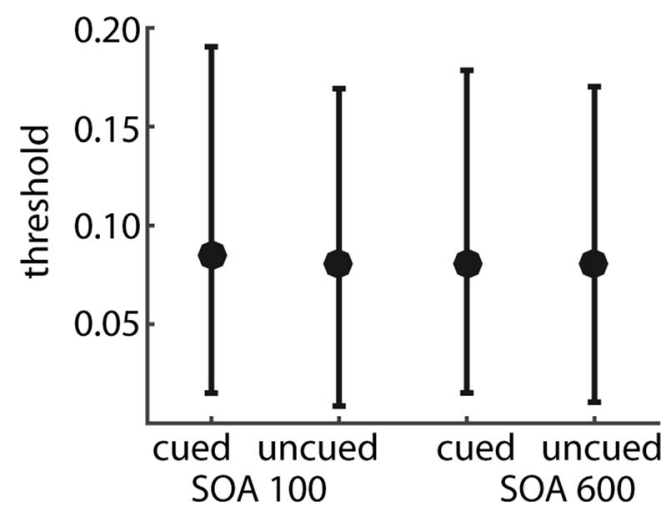

Fig. 5 Mean threshold and $95 \%$ confidence intervals (bootstraps) as a function of cueing condition and SOA 
failure to replicate. However, experiment 1 was designed to be a direct replication of Zimmermann et al. (2013) and also failed to find the effect. Note however that the power of the direct replication was matched to that of the initial study $(0.55)$ and therefore was underpowered. It remains possible that with more participants, the slow build-up of spatiotopic representations might emerge; then the difference with the conceptual replication would have to be explained. However, because the data, descriptively, did not show any trends towards such an effect, it seems an unlikely outcome.

A small error occurred in replicating the original design: the target dot diameter used in the present experiment was $0.2^{\circ}$, whereas in the original experiment it was $0.75^{\circ}$. This small difference in target size probably does not account for the difference of results. Indeed, saccadic suppression of displacement has been tested, and found, for various target sizes. There are variations in the amount of saccadic suppression between different reports (from approximately $30 \%$ in Bridgeman et al., 1975 to the 5-6 \% reported in Zimmermann et al., 2013, which is similar to the amount found in the current experiments), but they are not systematically related to target size. If saccadic suppression varies with target size, this modulation should affect overall performance and be orthogonal to the effect of pre-exposure duration.

The third experiment investigated the role of attention in spatiotopic performance, contrasting performance for an attended target versus unattended target. It is probably unfair to call the uncued condition "unattended," because a saccade was subsequently directed towards the uncued target. Because attention is oriented towards the target just before a saccade (Kowler et al, 1995; Deubel \& Schneider, 1996), attention was undoubtedly oriented to the saccade target in both cued and uncued conditions. The previous endogenous orientation of attention to the cued target did not confer any further advantage in the subsequent spatiotopic task. To fully address the question of whether only attended targets are remapped into spatiotopic coordinates (Joiner et al., 2011), future research will have to dissociate the saccade target from the attended target. The present results only suggest that the saccade target is probably always remapped because always attended.

Acknowledgments The research leading to these results has received funding from the Agence Nationale de la Recherche (Programme JCJC EPA) and European Research Council under the European Union's Seventh Framework Programme (FP7/2007-2013) / ERC grant agreement $n^{\circ}$ AG324070.

\section{References}

Brainard, D. H. (1997). The Psychophysics Toolbox. Spatial Vision, 10, 433-436.

Bridgeman, B., Hendry, D., \& Stark, L. (1975). Failure to detect displacement of the visual world during saccadic eye movements. Vision Research, 15, 719-722.

Cavanagh, P., Hunt, A. M., Afraz, A., \& Rolfs, M. (2010). Visual stability based on remapping of attention pointers. Trends in Cognitive Sciences, 14(4), 147-153.

Collins, T., Rolfs, M., Deubel, H., \& Cavanagh, P. (2009). Post-saccadic location judgments reveal remapping of saccade targets to nonfoveal locations. Journal of Vision, 9, 1-9.

Demeyer, M., De Graef, P., Wagemans, J., \& Verfaillie, K. (2010). Object form discontinuity facilitates displacement discrimination across saccades. Journal of Vision, 10(6), 17-17.

Deubel, H. (2004). Localization of targets across saccades: Role of landmark objects. Visual Cognition, 11, 173-202.

Deubel, H., Bridgeman, B., \& Schneider, W. (1998). Immediate postsaccadic information mediates space constancy. Vision Research, 38, 3147-3159.

Deubel, H., \& Schneider, W. (1996). Saccade target selection and object recognition: Evidence for a common attentional mechanism. Vision Research, 36, 1827-1837.

Deubel, H., Schneider, W., \& Bridgeman, B. (1996). Postsaccadic target blanking prevents saccadic suppression of image displacement. Vision Research, 36, 985-996.

Joiner, W. M., Cavanaugh, J., \& Wurtz, R. H. (2011). Modulation of shifting receptive field activity in frontal eye field by visual salience. Journal of Neurophysiology, 106(3), 1179-1190.

Jonikaitis, D., Szinte, M., Rolfs, M., \& Cavanagh, P. (2013). Allocation of attention across saccades. Journal of Neurophysiology, 109(5), $1425-1434$.

Kleiner, M., Brainard, D., \& Pelli, D. (2007). "What's new in Psychtoolbox-3?" Perception 36 ECVP Abstract Supplement.

Kowler, E., Anderson, E., Dosher, B., \& Blaser, E. (1995). The role of attention in the programming of saccades. Vision Research, 35, 1897-1916.

Niemeier, M., Crawford, J. D., \& Tweed, D. B. (2003). Optimal transsaccadic integration explains distorted spatial perception. Nature, 422, 76-80.

Pashler, H., \& Harris, C. R. (2012). Is the replicability crisis overblown? Three arguments examined. Perspectives on Psychological Science, 7(6), 531-536.

Pelli, D. G. (1997). The VideoToolbox software for visual psychophysics: Transforming numbers into movies. Spatial Vision, 10, 437-442.

Rolfs, M., Jonikaitis, D., Deubel, H., \& Cavanagh, P. (2011). Predictive remapping of attention across eye movements. Nature Neuroscience, 14(2), 252-256.

Sommer, M. A., \& Wurtz, R. H. (2008). Brain circuits for the internal monitoring of movements. Annual Review Neuroscience, 31, $317-338$.

Wexler, M. \& Collins, T. (2014). Orthogonal steps relieve saccadic suppression. Journal of Vision, 14(2):13, 1-9.

Zimmermann, E., Morrone, M. C., \& Burr, D. C. (2013). Spatial position information accumulates steadily over time. The Journal of Neuroscience, 33(47), 18396-18401. 